\title{
Levamisole-Adulterated Cocaine: A Case Series
}

\author{
Gerald W. Morris, Jr, MD, Bryan C. Mason, MD, Rachael Harris Sprunger, BS, \\ Holly Hake Harris, MD, Luke A. White, DO, and Dale A. Patterson, MD
}

The percentage of cocaine adulterated with levamisole is steadily increasing in the United States. In susceptible persons, this combination of drugs leads to a clinical syndrome that can include leukopenia and cutaneous manifestations. Many patients also demonstrate positive autoimmune markers. Biopsies of skin lesions often reveal a thrombotic pattern, leukocytoclastic vasculitis, or both. We report 2 cases of this clinical phenomenon, one of which was severe enough to require skin grafting. It is important for clinicians be aware of this emerging public health threat. (J Am Board Fam Med 2012;25:531-535.)

Keywords: Case Reports, Cocaine, Levamisole, Leukopenia, Purpura, Vasculitis

A number of adverse health effects are associated with illegal drug use. Complications of cocaine adulterated with levamisole were first reported in December 2009 by the Centers for Disease Control and Prevention, which suggested a link between cocaine use and unexplained agranulocytosis. ${ }^{1}$ More recently there have been reports of cocaine users presenting with retiform (net-like or reticulated) purpuric lesions and white blood cell suppression including leukopenia or neutropenia. ${ }^{2-6}$ We present 2 additional cases of otherwise unexplained leukopenia and retiform purpuric lesions in patients who tested positive for cocaine.

\section{Case 1}

A 51-year-old African-American woman presented to the emergency department with a 1-day history of multiple, exquisitely tender retiform purpuric papules and plaques that began on her ears and lower face and progressed to her extremities (Figure 1A and B). Her medical history was

This article was externally peer reviewed.

Submitted 10 October 2011; revised 18 February 2012; accepted 27 February 2012.

From the Memorial Family Medicine Residency (GWM, BCM, DAP) and the Department of Internal Medicine (LAW), Memorial Hospital, South Bend, IN; Midwestern University, Chicago, IL (RHS); South Bend Clinic, South Bend, IN (HHH); and Indiana University School of Medicine, South Bend, IN (HHH).

Funding: none.

Conflict of interest: none declared.

Corresponding author: Dale A. Patterson, MD, Memorial Hospital, Family Medicine Residency Program, 714 North Michigan Street, South Bend, IN 46601 (E-mail: dpatterson@memorialsb.org). significant for anemia, hepatitis $\mathrm{C}$, hematuria, hypertension, chronic cocaine abuse, and hospitalization 7 months earlier for auricular ischemia thought to be caused by cocaine use. Previous autoimmune evaluation was significant for equivocal double-stranded DNA antibodies on 2 occasions, marginally elevated rheumatoid factor on 2 of 4 occasions, and elevated histone antibodies at $7.7(0-1.0)$ during the hospitalization for auricular ischemia. She denied using anticoagulants including warfarin. Family history revealed lupus in a maternal uncle.

At presentation, complete blood count demonstrated a white blood cell count (WBC) of $3600 / \mu \mathrm{L}$ (normal range, $4000-11,000 / \mu \mathrm{L}$ ), sedimentation rate of $97 \mathrm{~mm} / \mathrm{hr}$ (normal range, 0-30 mm/hr), and a urine drug screen positive only for cocaine. HIV, hepatitis A, and hepatitis $\mathrm{B}$ screens were negative. Hepatitis $\mathrm{C}$ was confirmed. Serum cryoglobulins and antineutrophil antibody were negative. Transthoracic echocardiogram demonstrated no vegetation or mass. Skin pathology confirmed a thrombotic vasculitis with fibrin thrombi noted in the deep and superficial blood vessels (Figure 2A and B). A subsequent coagulation workup revealed an elevated lupus anticoagulant 1 at 60.1 seconds (normal, $<45$ seconds). Four days into her hospitalization, the patient's hemoglobin dropped from 10.5 $\mathrm{g} / \mathrm{dL}$ (level at presentation) to $7.3 \mathrm{~g} / \mathrm{dL}$. Laboratory evaluation for hemolytic anemia was unremarkable. A peripheral blood smear demonstrated severe normocytic anemia with rare 
Figure 1. Tender retiform purpuric papules and plaques on left ear (A) and leg (B) that developed after using cocaine suspected of being adulterated with levamisole.

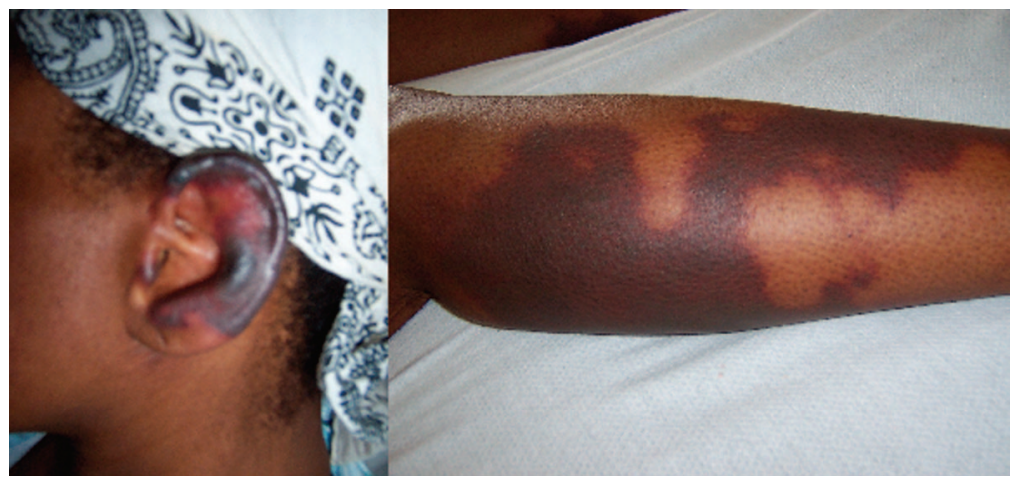

schistocytes and microspherocytes and a granulocytic left shift with toxic changes. She received 2 units of packed red blood cells and had no further decline in hemoglobin.

During the course of her hospitalization, tense bullae formed over the pretibial area. There were no petechiae or oral lesions. Blood cultures were negative, sputum culture grew Haemophilus influenza, and urine culture grew Escherichia coli. Antibiotic treatment was initiated. Sedimentation rate was $90 \mathrm{~mm} / \mathrm{hr}$ on day 5 and $55 \mathrm{~mm} / \mathrm{hr}$ on day before discharge (day 6). Meanwhile, her WBC increased to 10,200. At discharge, outpatient wound care was arranged and oral pain medications were provided.

\section{Case 2}

A 23-year-old white woman presented to the emergency department with a 4-day history of painful lesions located on her face, trunk, and extremities. These lesions included widespread retiform purpuric coalescing plaques on her auricular helices and a prominent pattern of branching livedoid plaques with well-demarcated borders (Figure 3A and $\mathrm{B}$ ). The patient recounted similar but less severe lesions when hospitalized 1 year earlier. Medical history was significant for anemia, osteomyelitis of her left foot, and chronic cocaine abuse. At presentation, her WBC count was $2600 / \mu \mathrm{L}$, sedimentation rate was $109 \mathrm{~mm} / \mathrm{hr}$, and a urine drug screen was positive for cocaine. Her previous hospitalization for a similar presentation also was notable for leukopenia, an elevated sedimentation rate, and positive urine drug screen for cocaine. She was also antineutrophil antibody positive but was negative for SSA/Ro, SSB/La, RNP, scleroderma, JO-1, and antismooth muscle antibodies. During the current hospitalization, hepatitis and HIV serology were negative. Blood cultures were negative. The lesions blistered but steadily improved, so the patient was discharged after a 3-day hospitalization with wound care and oral pain medications. At discharge, her WBC count was 3300/ $\mu \mathrm{L}$.

Eleven days later the patient was readmitted to the hospital. Her WBC count was 2000/ $\mu$ L, sedimentation rate was $>120 \mathrm{~mm} / \mathrm{hr}$, and urine drug

Figure 2. Punch biopsies taken from case 1 demonstrated fibrin thrombi located in the deep and superficial blood vessels.

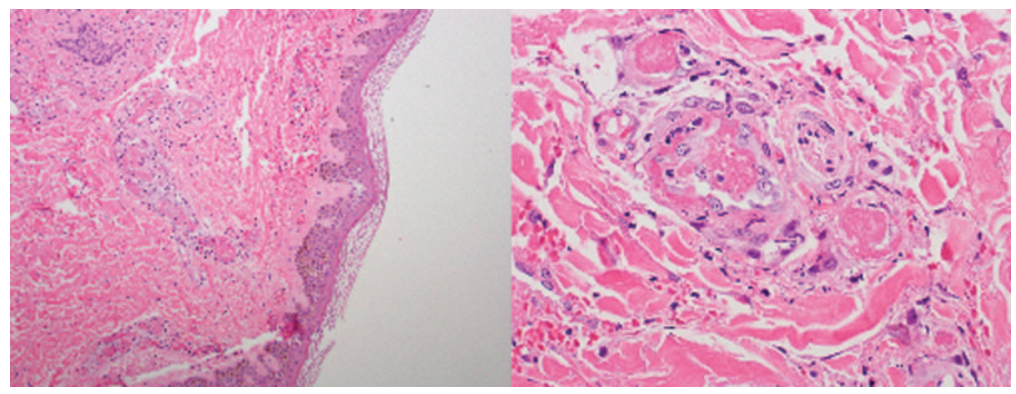


Figure 3. Painful branching livedoid plaques with well demarcated borders on legs (A) and retiform purpuric coalescing plaques on left ear (B). These lesions developed days after using cocaine suspected of being adulterated with levamisole.

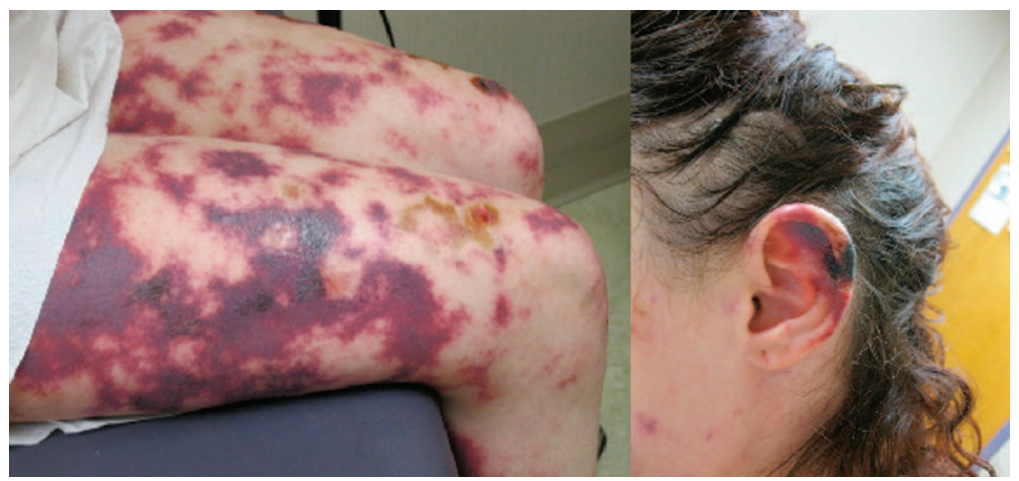

screen was again positive for cocaine. The lesions on her ears had progressed with the development of bullae, central necrosis, and peripheral erythema. She ultimately developed full-thickness necrosis (Figure 4A), defined by the formerly purpuric plaque margins. Blood cultures were negative but a wound culture grew out Staphylococcus aureus, which was resistant to methicillin. Her hemoglobin fell from $8.3 \mathrm{~g} / \mathrm{dL}$ to $6.4 \mathrm{~g} / \mathrm{dL}$. She was subsequently transfused with 2 units packed red blood cells. Given the extent of the patient's advancing wounds, she was transferred to a regional burn center for further treatment and care.

Approximately 2 months after receiving skin grafts at a regional burn center, the patient presented to the emergency department with a reoccurrence of lesions after using cocaine 3 to 4 days earlier. Her WBC count was 3800/ $\mathrm{LL}$. A drug screen on the day after presentation confirmed re- peat cocaine use. She again experienced acute anemia, which responded to packed red blood cells. Wound cultures grew polymicrobial flora and the patient was treated with antibiotics. Her lesions were extensive and some encroached on the previously grafted skin sites (Figure 4B), necessitating transfer back to the regional burn center for definitive management.

\section{Discussion}

Originally used as an antihelminthic agent, levamisole found a role in medicine as an immunomodulator to treat autoimmune disorders such as rheumatoid arthritis and nephrotic syndrome. In the early 1990s, it was approved as an adjuvant therapy in the treatment of colon cancer. However, it was voluntarily withdrawn from the US market because newer chemotherapeutic agents were more effec-

Figure 4. Full-thickness dermal necrosis with sloughing in a wound that was formerly defined by a purpuric plaque (A). After receiving skin grafts, the patient returned 2 months later with necrotic skin lesions encroaching on the previously grafted sites after reusing cocaine (B).

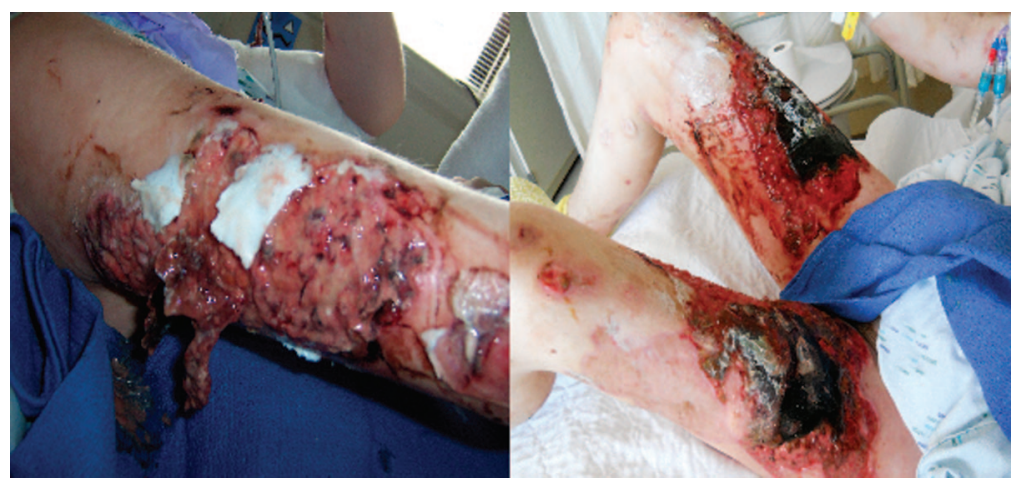


tive and less toxic. Today, levamisole continues to be used as a veterinary antihelminthic agent. Since it was first recognized by the US Drug Enforcement Administration in 2003, ${ }^{7}$ the incidence of cocaine adulterated with levamisole has been increasing. In 2009, 69\% of all cocaine seized by United States Drug Enforcement Administration was found to contain levamisole. ${ }^{1}$ Recently, Buchanan et $\mathrm{al}^{8}$ reported that $78 \%$ of urine samples from a series of hospitalized patients who tested positive for cocaine also tested positive for levamisole. The exact purpose for adding levamisole to cocaine is unknown but it has been postulated that levamisole has a potentiating effect on cocaine through the inhibition of catecholamine breakdown. ${ }^{9}$ Levamisole also may be added to cocaine because of its stimulant effects, its ability to pass street tests of purity, its similarity to cocaine in appearance, and its ability to bulk up crack. ${ }^{10}$

As early as the 1970s, case reports of levamisoleinduced skin reactions or neutropenia were documented. ${ }^{11,12}$ Recent case reports of patients thought to be affected by cocaine adulterated with levamisole present with a similar clinical picture: urine drug screen positive for cocaine, leukopenia, characteristic cutaneous manifestations, and positive autoimmune serologies. With more severe leukopenia, patients may have infectious complications as well. The cutaneous manifestations often are described as a retiform purpura. ${ }^{4}$ The rash can occur anywhere on the body but has a predilection for the extremities and ears. Various autoimmune markers may remain for 2 to 14 months after discontinuation of the drug. ${ }^{13}$ The reason for this is uncertain. Published cases report a higher incidence of women affected. Patients with the human leukocyte antigen B27 genotype have been found to have a greater risk for agranulocytosis, ${ }^{14}$ but neither patient presented here was tested for human leukocyte antigen B27.

Histopathologic patterns often include some degree of thrombosis, leukocytoclastic vasculitis, or both, ${ }^{2-6,13}$ making biopsy useful in narrowing the differential diagnosis. Urinary detection of levamisole is a valuable tool as well. However, levamisole has a half-life of approximately 5.6 hours, ${ }^{15}$ making its detection in urine difficult. Neither of the patients discussed in this article were tested for levamisole. A liquid chromatography-tandem mass spectrometry method for detecting urinary levamisole recently has been developed, but to date there have been no randomized trials evaluating its use. ${ }^{16}$
Therefore, the diagnosis of cutaneous vasculitis with retiform purpura in the setting of cocaine adulterated with levamisole often remains a diagnosis of exclusion.

Interestingly, each of our cases had prior hospitalizations with similar lesions, though less extensive; both also had presented in the past with leukopenia and urine drug screens positive for cocaine. Their worsening presentations could suggest an autoimmune phenomenon or represent the effects of increasing exposure to levamisole. During their hospitalization, both patients demonstrated an increased WBC count and their lesions did not progress when not using cocaine.

\section{Conclusions}

Leukopenia in conjunction with retiform purpura and a positive cocaine screen is quickly becoming a recognizable clinical syndrome. In many cases this diagnosis cannot be made definitively. A high index of suspicion must be maintained-and testing for levamisole considered as soon as possible-if this is considered a potential cause for a patient's clinical presentation. Health care providers need to be aware of this potential community health threat as more and more cases are discovered in conjunction with the increasing rate of levamisole adulterated cocaine.

The authors would like to thank Chuck LeGuern for his library services.

\section{References}

1. Centers for Disease Control and Prevention. Agranulocytosis associated with cocaine use-four states, March 2008-November 2009. MMWR Morb Mortal Wkly Rep 2009;58:1381-5.

2. Gross R, Brucker J, Bahce-Altuntas A, et al. A novel cutaneous vasculitis syndrome induced by levamisole-contaminated cocaine. Clin Rheumatol 2011; 30:1385-92.

3. Chung C, Tumeh P, Birnbaum R, et al. Characteristic purpura of the ears, vasculitis, and neutropenia-a potential public health epidemic associated with levamisole-adulterated cocaine. J Am Acad Dermatol 2011;65:722-5.

4. Geller L, Whang T, Mercer S, Phelps R. Retiform purpura: a new stigmata of illicit drug use? Dermatology Online J 2011;17:7.

5. Waller J, Feramisco J, Alberta-Wszolek L, McCalmont T, Fox L. Cocaine-associated retiform purpura and neutropenia: is levamisole the culprit? J Am Acad Dermatol 2010;63:530-5. 
6. Ullrich K, Koval R, Koval E, Bapoje S, Hirsh J. Five consecutive cases of a cutaneous vasculopathy in users of levamisole-adulterated cocaine. J Clin Rheum 2011;17:193-6.

7. Valentino A, Fuentecilla K. Levamisole: an analytical profile. Microgram J 2005;3:134-7.

8. Buchanan J, Heard K, Burbach C, Wilson M, Dart R. Prevalence of levamisole in urine toxicology screens positive for cocaine in an inner-city hospital. JAMA 2011;305:1657-8.

9. Chang A, Osterloh J, Thomas J. Levamisole: a dangerous new cocaine adulterant. Clin Pharmacol Ther 2010;88:408-11.

10. Kiley B. The mystery of the tainted cocaine. The Stranger 2010 August 17. Available from: http://www. thestranger.com/seattle/Content? mode $=$ print\&oid $=$ 4683741. Accessed May 7, 2012.

11. MacFarlane D, Bacon P. Levamisole-induced vasculitis due to circulating immune complexes. BMJ 1978;1:407-8.
12. Scheinberg M, Bezerra J, Almeida F, Silveira L. Cutaneous necrotising vasculitis induced by levamisole. BMJ 1978;1:408.

13. Rongioletti F, Ghio L, Ginevri F, et al. Purpura of the ears: a distinctive vasculopathy with circulating autoantibodies complicating long-term treatment with levamisole in children. Br J Dermatol 1999;140:948-51.

14. Czuchlewski D, Brackney M, Ewers C, et al. Clinicopathologic features of agranulocytosis in the setting of levamisole-tainted cocaine. Am J Clin Pathol 2010;133:466-72.

15. Kouassi E, Caille G, Lery L, Lariviere L, Vezina M. Novel assay and pharmacokinetics of levamisole and p-hydroxylevamisole in human plasma and urine. Biopharm Drug Dispos 1986;7:71-89.

16. Lynch K, Dominy S, Graf J, Kral A. Detection of levamisole exposure in cocaine users by liquid chromatography-tandem mass spectrometry. J Analytical Toxicol 2011;35:176-8. 